九州大学学術情報リポジトリ

Kyushu University Institutional Repository

\title{
Experimental Study on Effect of Spires on the Lateral Nonuniformity of Mean Flow in a Wind Tunnel
}

Rahmat, Nurizzatul Atikha

Graduate School of Engineering, Kyushu University

Hagishima, Aya

Faculty of Engineering Sciences, Kyushu University

Ikegaya, Naoki

Faculty of Engineering Sciences, Kyushu University

谷本, 潤

Faculty of Engineering Sciences, Kyushu University

https://doi.org/10.5109/1929670

出版情報: Evergreen. 5 (1)，pp.1-15，2018-03. Green Asia Education Center バージョン：

権利関係 : 


\title{
Experimental Study on Effect of Spires on the Lateral Nonuniformity of Mean Flow in a Wind Tunnel
}

\author{
Nurizzatul Atikha Rahmat ${ }^{1, *}$, Aya Hagishima ${ }^{2}$, Naoki Ikegaya ${ }^{2}$, Jun Tanimoto ${ }^{2}$ \\ ${ }^{1}$ Graduate School of Engineering Sciences, Kyushu University, Japan \\ ${ }^{1}$ University Malaysia Pahang, Malaysia \\ ${ }^{2}$ Faculty of Engineering Sciences, Kyushu University, Japan \\ *Author to whom correspondence should be addressed, \\ E-mail: ezatikha@gmail.com
}

(Received November 30, 2017; accepted January 14, 2018)

\begin{abstract}
The present work discusses in detail the experimental settings to establish a thick artificially generated urbanlike atmospheric boundary layer in a short-fetch-length wind tunnel. It summarizes the comprehensive review of past studies on the flow behind quarter-elliptic-wedge spires, and clarifies the current issue of the spire setting in a wind tunnel experiment. This study investigates the persistent spanwise heterogeneity, the spatial structures of the flow past a row of quarter-elliptic-wedge spires, the streamwise distance required for a wake of elliptic-wedge spires to naturally recover, and how the mean flow changes farther downstream. Two experimental conditions were implemented in the present work (without and with spires) to analyse the effect of mean flow heterogeneity both within and above boundary layer height (BLH) regions, developing over a smooth wall that was produced by the installation of a flat plate on the wind tunnel floor.
\end{abstract}

Keywords: wind tunnel, spire, wake flow, wall shear boundary layer.

\section{Nomenclature}

$\mathrm{RSD}_{U} \quad$ : Relative standard deviation (\%)

$S \quad$ : Height of spires (m)

$s \quad$ : Spires lateral spacing $(\mathrm{m})$

$U \quad$ : Streamwise velocity $(\mathrm{m} / \mathrm{s})$

$U_{n} \quad$ : Normalized velocity (-)

$U_{\text {ref }} \quad$ : Reference velocity (m/s)

WO : Without spire case

WS : With spire case

$x \quad$ : Streamwise distance (m)

y $\quad$ : Lateral distance (m)

$y_{S} \quad:$ Width of spires (m)

z $\quad$ : Vertical distance (m)

$\delta \quad$ : Boundary layer height (BLH) $\sigma \quad$ : Standard deviation of velocity $(\mathrm{m} / \mathrm{s})$

$v \quad:$ Kinematic viscosity $\left(\mathrm{m}^{2} / \mathrm{s}\right)$

\section{Introduction}

Nowadays, in corresponds to the rapid economic growth, high birth rate and migration, the number of megacities are increasing globally. Especially in Asian countries with more than half of its population residing in cities (United Nations 2016 ${ }^{1}$ ). In order to accommodate such dense urban population, Asian megacities are comprised with numerous skyscrapers and tall buildings, geometries that are much different from conventional western cities.

In such rising megacities, the urban environmental degradation associated with the atmospheric features affected by densely packed tall buildings has become a social issue. For example, the urban heat island (UHI) phenomenon (Oke 1987²) has caught major attention for a couple of decades. In 
addition to UHI, air pollution emitted from urban activities has increased risk of respiratory. In order to establish countermeasure seeking for green and sustainable urban environment, detailed understanding on the aerodynamic nature of urban boundary layer which govern the transport process of heat, moisture and pollutants is important.

Under these circumstances, numerous studies on the flow nature of urban atmosphere, have been intensively conducted as an essential factor in determining the safety, health, and comfort of urban habitants as reviewed by Blocken and Carmeliet2003 ${ }^{3)}$ ), Grimmond 20064), Kanda 20065), Aly 20146). Since the flow state of an urban atmosphere is mostly turbulent owing to both the mechanical shear created by the urban roughness and the buoyancy production caused by surface heating, the prognostic equations of velocity, temperature, and scalar concentrations cannot be directly solved (e.g., Stull 19887). Hence, some approximation and simplification of the phenomenon are required. This fact coincides with the importance of numerous field observations and scaled model experiments such as wind tunnel experiments in the research of the wind engineering. This study focuses on one of the basic experimental techniques necessary to reproduce a thick urban-like atmospheric boundary layer in a relatively small wind tunnel.

Scaled model experiments in a wind tunnel have significantly contributed to deepening the understanding of the effects of urban roughness on transport processes (Cermak and Arya 19708). Meanwhile, within the urban surface layer, surface drags due to urban obstacles and the buoyancy force associated with the diurnal cycle of atmospheric stability and surface thermal forcing continuously produce turbulence on various spatial and temporal scales. By contrast, it has been known that the turbulence of the flow artificially generated inside a wind tunnel is generally much weaker compared to the real urban atmosphere because there is no source to sustain the turbulence, unlike the real urban atmosphere (e.g. Counihan 19739).

Furthermore, creating a fully developed boundary layer with sufficient depth in a wind tunnel is always challenging because of the limited streamwise distance of the tunnel. Therefore, intensive efforts to reproduce quasi-urban turbulent flow inside a wind tunnel have been conducted for decades. Among numerous attempts, multiple fans (Nishi et al. 199710) or the installation of some obstacles at the upwind region of a wind tunnel (such as fences, mesh screens ${ }^{11)}$, grids ${ }^{12)}$, an array of cylindrical rods ${ }^{13)}$, or rectangular bars ${ }^{13)}$ ) has been widely applied. In particular, the use of slender vortex generators called spires is one of the most acknowledged practices. Figure 1 illustrates typical configurations of spires: triangular spire (Armitt and Counihan 1968 ${ }^{14}$ ), triangular and cranked spire (Counihan 1969 ${ }^{15)}$ ), triangular with splitter-plate spire (Irwin 19816), quarter-plane elliptic spire (Counihan 196915), and quarter-elliptic-wedge spire (Counihan 1969 ${ }^{15)}$, Counihan et al. 1974 ${ }^{17)}$ ).
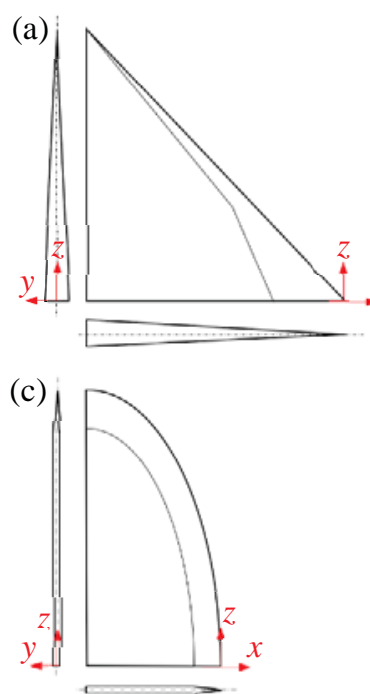

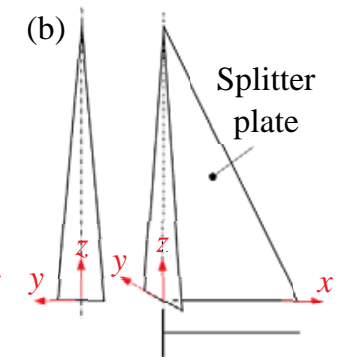

(d)

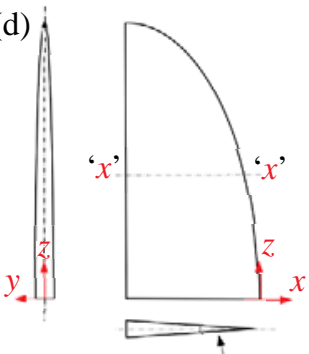

Wedge angle is constant at any horizontal section ' $x-x$ '.
Fig. 1: Schematic figures of (a) triangular and cranked, (b) triangular with splitter plate, (c) quarterplane-elliptic, and (d) quarter-elliptic-wedge spire. Axes $x, y$, and $z$ refer to streamwise, lateral, and vertical directions.

In most wind tunnel experiments, these slender obstacles are installed on the windward side of a test section of a wind tunnel in a spanwise direction with a regular interval. The wake behind each spire is characterized by a region of strong velocity deficit as well as large vortices. This causes a strong heterogeneous mean flow and gradually merges with the wall boundary layer over the tunnel floor, resulting in a new structure which resembles a thick atmospheric boundary layer.

Despite the popularity of spires, a comprehensive knowledge of the flow features past multiple spires has 
not been well compiled, and knowledge of the arrangement of spires has been shared only inside each laboratory.

Under these circumstances, the objectives of this paper are to provide a comprehensive review and comparison of past experiments on the flow behind spires, and clarify the knowledge gap in the current studies of the spire setting, which will be given in chapter 2. In addition, based on the knowledge gap shown in chapter 2, this study intends to grasp the three-dimensional structure of the flow past spires based on our wind tunnel experiment as described in chapter 3. Based on this experimental result, the necessary length between spires and measurement positions is clarified in chapter 4 .

\section{Past studies on spires}

\subsection{Basic strategies of spire setting}

The fundamental idea of spires to reproduce a deep quasi-atmospheric boundary layer in a wind tunnel with a limited streamwise length was presented by Armitt and Counihan $1968^{14)}$ and Counihan 1969 ${ }^{15)}$.

Counihan $1969^{15)}$ scrutinized the flow past spires with four different shapes: triangular, cranked triangular, plane quarter elliptic, and quarter elliptic wedge. Meanwhile, Irwin $1981^{16)}$ presented a chart which determines the proper dimension of a triangular spire with a splitter plate according to the target velocity profile expressed by the power law $\left[U / U_{\infty}=\right.$ $\left.(y / \delta)^{a}\right]$.

After these pioneering studies, numerous wind tunnel experiments used spires to generate deep boundary layer with strong turbulence based on the methods suggested by Counihan 1969'15) and Irwin 19816). Table 1 lists literature that investigated the characteristics of the flow modulated by spires under various spire settings.

Figure 2 shows the boundary layer height (BLH) over smooth and rough surfaces at various fetch distances under the conditions of with and without spires observed by past experiments. The figure illustrates the effectiveness of spires in deepening the boundary layer. The present experimental data are also included in Fig. 2(a), which will be discussed later in sections 3 and 4 in a comprehensive examination of the wake structures of spires. In addition, the equation of the boundary layer depth, $\delta(x)=0.38\left(\frac{U_{\infty} x}{v}\right)^{-1 / 5} x$, derived from the power-law velocity profile over a smooth wall, the Blasius profile and Karman's momentum equation are adopted as a reference.

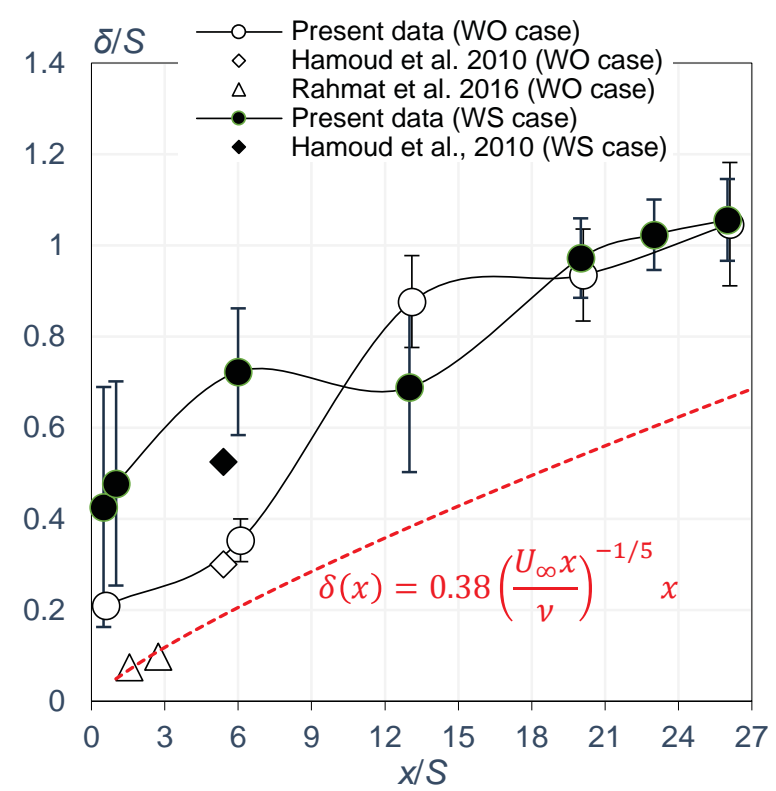

(a) Smooth surface

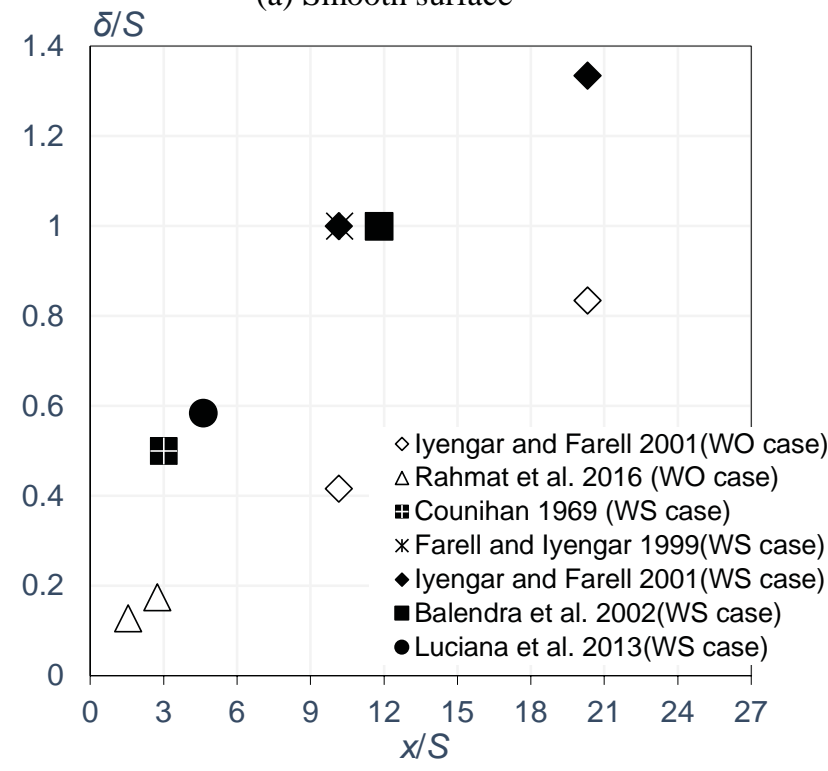

(b) Rough surface

Fig. 2: Boundary layer height normalized by spire height against streamwise distance over (a) smooth and (b) rough surfaces in wind tunnels observed by various experiments including present study. WS and WO refer to conditions with and without spire. Dashed line refers to equation of boundary layer depth over smooth wall. 
As shown in Fig. 2(a), the BLH measured at the near-wake region of a case without a spire given by Rahmat et al. 2016 ${ }^{31)}$ is consistent with the theoretical equation curve of the boundary layer depth over a smooth wall. However, the other two experiments show discrepancies which were probably caused by the effect of the inflow condition. By contrast, the BLHs over rough surfaces with and without spires [Fig. 2(b)] obtained by different experiments show similar trends. Furthermore, the BLHs that are past spires are larger than those without spires by about $32 \%$ in average.

It is noteworthy that in most experiments the fetch area between the spires and the measurement position is covered by roughness, such as an array of blocks or rods. There is little experimental data of the flow over a smooth surface with a fetch longer than $5.4 S$ ( $S$ refers to the height of the spires) downwind from the spires except in the present study. This may be because the spire effect for generating a deep boundary layer is limited for a relatively short fetch length if the fetch area is smooth, as Armitt and Counihan $1968^{5)}$ pointed out.

\subsection{Effect of spire shape on BLH}

The four types of spire shape shown in Fig. 2 were proposed by Counihan 1969 ${ }^{15}$. Two of them, i.e. quarter-elliptic wedge and triangular, have been mainly used.

The excellence of quarter-elliptic-wedge spires was first reported by Counihan 1969 ${ }^{15}$ ), which shows that this shape can realize the deepest BLH and relatively good uniformity in a lateral direction compared with the other three shapes. Irwin 19816) and Farell and Iyengar 199923) suggested that the BLH developed by elliptical spires is $20 \%$ deeper than that produced by triangular spires with an equivalent height.

\subsection{Heterogeneity of the mean flow caused by spires}

Although spires are effective in generating deep quasi-atmospheric boundary layers in a wind tunnel with a limited streamwise length, the heterogeneity of the mean flow caused by the wake of each spire has been a longstanding issue since a study by Armitt and Counihan $1968^{14)}$. In general, larger spires are effective in obtaining a deep BLH. However, smaller ratios of the fetch length to the spire height $x / S$ cause strong heterogeneity of the mean flow. By contrast, a larger $x / S$ can improve this heterogeneity. However, the turbulent intensity becomes weak. Therefore, proper layout designs of spires to achieve both strong turbulent intensity and negligible lateral heterogeneity of the flow are essential. Thus, several studies have investigated the effects of the type of spire shape and the detailed design of spires and the lateral interval between spires.

For example, Counihan 1969 ${ }^{15}$ ) reported that spires of quarter-elliptic-wedge shape with a $5^{\circ}$ wedge angle must be positioned closer than that with a $6^{\circ}$ angle in order to obtain a similar level of lateral uniformity of the wake flow without requiring a reduction in the turbulence intensity and a modification of the power spectral of the velocity. Irwin $1981^{16)}$ pointed out that triangular spires with a lateral spacing of $S / 2$ can produce sufficient spatial uniformity of the mean velocity at a $6 S$ fetch length.

Recently, Hohman et al. 2015 ${ }^{30}$ reported that the spanwise uniformity can be improved by using a shorter interval of spires $(0.3 S)$ and smaller wedge angle $\left(3^{\circ}\right)$. The same level of uniformity $(1.2 \%)$ was achieved at a fetch length of $1.5 \mathrm{~S}$ compared to the original design of Counihan 196915), i.e. an interval of $0.6 S$ and angle of $6^{\circ}$ measured at $3 S$.

By contrast, Rahmat et al. 2016 ${ }^{31)}$ investigated the wake structure past a single quarter-elliptic-wedge spire with a semi wedge angle of $5.7^{\circ}$ for both smooth and rough surface conditions. The noted that the wake generated by the spire remains at a fetch length of 2.7 $S$, and that the heterogeneity of the mean flow is more dominant within a boundary layer compared to the above boundary layer.

Even though these former studies provide useful guidelines to determine the shape and lateral interval of spires for creating flow with better uniformity, comprehensive review of the flow structures generated by a row of spires itself has not been well highlighted. In principle, the enhancement effects of spires on both the BLH and the turbulent intensity are a result of the mean wind shear and vortices created by the wake of each spire, which extends up to the height of the spires. Thus, improving the spanwise heterogeneity of the mean flow past spires is a difficult challenge.

\subsection{Objectives}

Under these circumstances, the present study focuses on the recovery process of wake flows created by spires over a wall boundary layer based on the 


\begin{tabular}{|c|c|c|c|c|c|c|c|c|c|c|c|c|c|c|}
\hline & 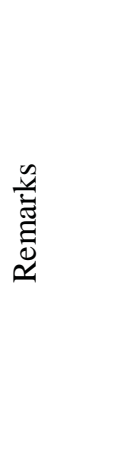 & 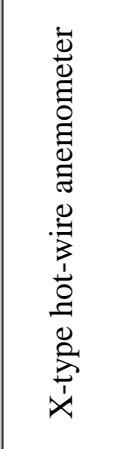 & & & 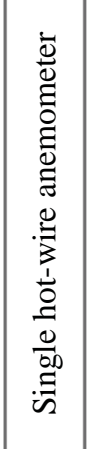 & ' & & 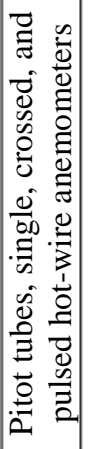 & 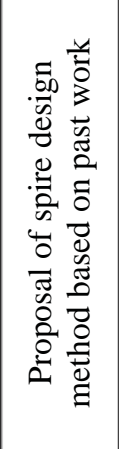 & 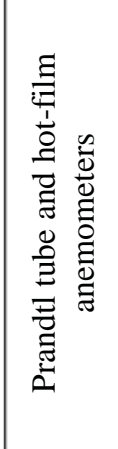 & 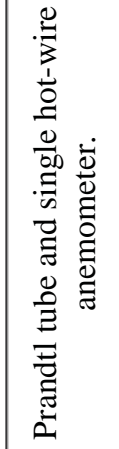 & 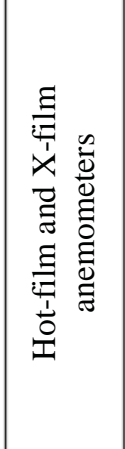 & 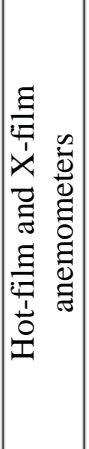 & 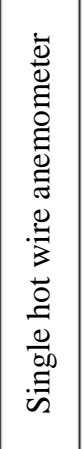 \\
\hline & 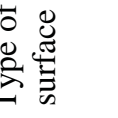 & 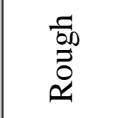 & 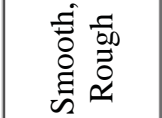 & 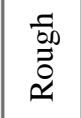 & 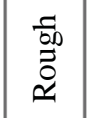 & ' & & 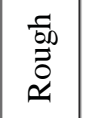 & I & $\begin{array}{l}\bar{~} \\
\overline{0} \\
\approx\end{array}$ & $\begin{array}{l}\overline{0} \\
\overline{0} \\
\overline{0}\end{array}$ & $\begin{array}{l}\overline{0} \\
\overline{0} \\
\overline{0}\end{array}$ & 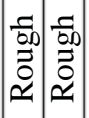 & 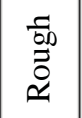 \\
\hline & 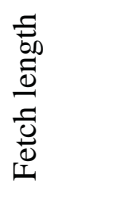 & 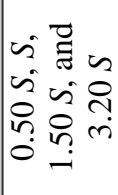 & 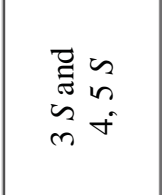 & & $\begin{array}{l}\text { w } \\
\text { ln } \\
\text { ln }\end{array}$ & ' & 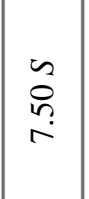 & $\begin{array}{l}n \\
\text { in }\end{array}$ & w & I & \begin{tabular}{l}
$n$ \\
\multirow{-}{*}{}
\end{tabular} & \begin{tabular}{l} 
w \\
\multirow{1}{*}{} \\
0
\end{tabular} & 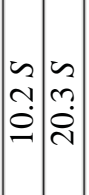 & ' \\
\hline 斿 & $\underset{3}{5}$ & $\begin{array}{l}\infty \\
\infty \\
\infty \\
\dot{m}\end{array}$ & y & $\tilde{m}$ & w & s & $\begin{array}{l}\text { un } \\
\text { ஸn } \\
\text { in }\end{array}$ & $\begin{array}{l}n \\
0 \\
\tilde{m} \\
\tilde{\omega}\end{array}$ & $I$ & $\tilde{N}$ & $\stackrel{\sim}{\sim}$ & $\begin{array}{l}\text { w } \\
\text { qu } \\
-i\end{array}$ & 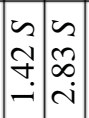 & $\begin{array}{l}n \\
o \\
+ \\
\dot{q}\end{array}$ \\
\hline 泀 & 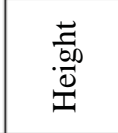 & $\begin{array}{l}\text { s } \\
\stackrel{\sim}{N} \\
\rightarrow\end{array}$ & \begin{tabular}{l}
$\tilde{w}$ \\
\multirow{\sim}{r}{}
\end{tabular} & $m$ & 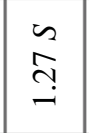 & $\omega$ & $\begin{array}{l}\text { w } \\
\text { m } \\
\text { - }\end{array}$ & $\begin{array}{l}n \\
0 \\
\text { m. } \\
\text { in }\end{array}$ & $I$ & $\underset{m}{m}$ & $\underset{m}{m}$ & $\begin{array}{l}\text { w } \\
\text { ํㅜㄹ } \\
\text { hr }\end{array}$ & 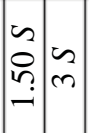 & $\begin{array}{l}\tilde{a} \\
\tilde{a} \\
\end{array}$ \\
\hline & 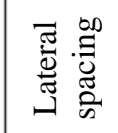 & s & $\begin{array}{l}\text { w } \\
\text { ํㅜ } \\
\text { 10 }\end{array}$ & $\begin{array}{l}\text { w } \\
\text { 10 } \\
\text { 10 }\end{array}$ & ' & $\begin{array}{l}\text { w } \\
\stackrel{2}{0} \\
0\end{array}$ & & ' & $\begin{array}{l}\text { nos } \\
\text { ำ } \\
\text { 10 }\end{array}$ & $\begin{array}{l}\text { w } \\
0 \\
0 \\
0\end{array}$ & $\begin{array}{l}\text { n } \\
\text { 10 } \\
0\end{array}$ & ' & & $\begin{array}{l}\text { w } \\
0 \\
\text { ڤn. } \\
0\end{array}$ \\
\hline 氙 & 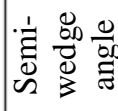 & ' & 8 & ¿ & ¿ & ' & $\begin{array}{l}\stackrel{\circ}{\stackrel{1}{n}} \\
\text { in }\end{array}$ & ' & ' & 1 & ' & ' & 80 & ¿ \\
\hline 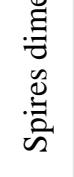 & 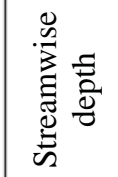 & ' & $\begin{array}{l}\text { w } \\
\text { เి } \\
0\end{array}$ & $\begin{array}{l}\text { w } \\
0 \\
\text { เִ } \\
0\end{array}$ & $\begin{array}{l}\text { us } \\
0 \\
\text { Hุ } \\
0\end{array}$ & \begin{tabular}{l}
$u$ \\
\multirow{J}{*}{} \\
$\vdots$ \\
0
\end{tabular} & $\begin{array}{l}\text { w } \\
0 \\
\text { เִ } \\
0\end{array}$ & ' & ' & 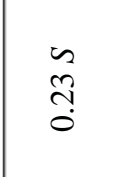 & & ' & \begin{tabular}{l|l}
$w$ & $s$ \\
0 & 0 \\
$\vdots$ & 0 \\
0 & 0 \\
0
\end{tabular} & ' \\
\hline & $\frac{\bar{E}}{3}$ & ' & ' & 1 & ' & 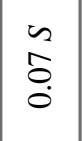 & ' & ' & 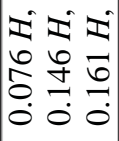 & \begin{tabular}{l}
$n$ \\
\multirow{0}{*}{} \\
$\stackrel{1}{0}$ \\
0
\end{tabular} & & ' & & $\begin{array}{l}n \\
ت \\
ت \\
0\end{array}$ \\
\hline & 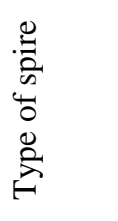 & 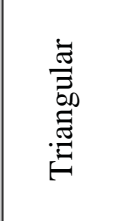 & 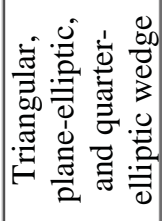 & 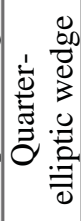 & 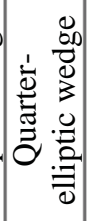 & 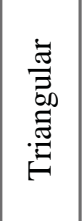 & 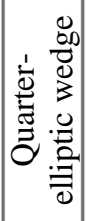 & 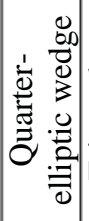 & 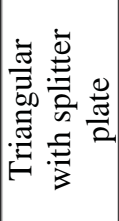 & 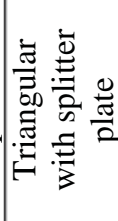 & 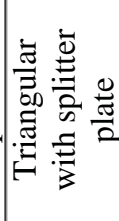 & 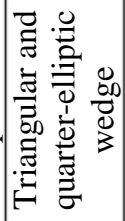 & 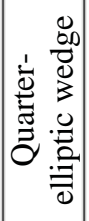 & 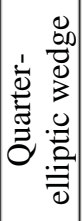 \\
\hline & 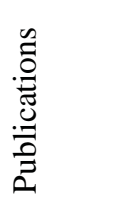 & 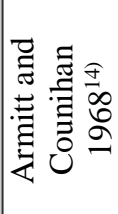 & 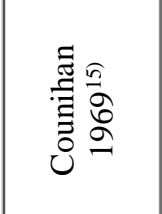 & 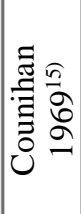 & 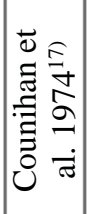 & 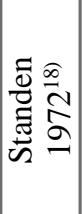 & 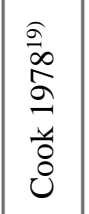 & 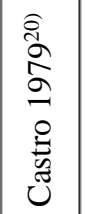 & 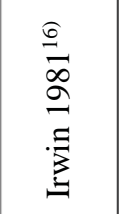 & 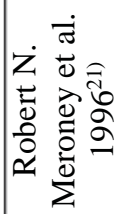 & 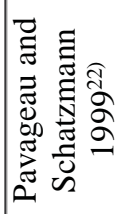 & 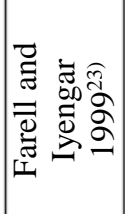 & 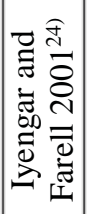 & 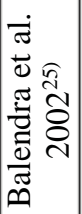 \\
\hline
\end{tabular}




\begin{tabular}{|c|c|c|c|c|c|}
\hline 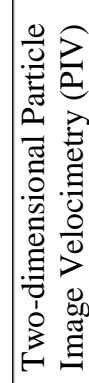 & 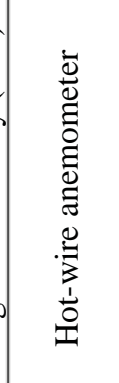 & 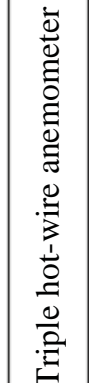 & 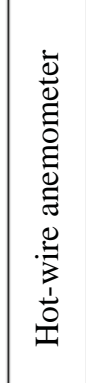 & 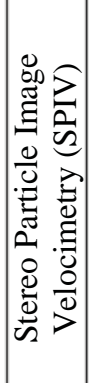 & 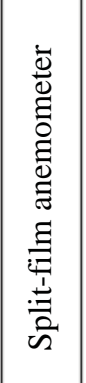 \\
\hline 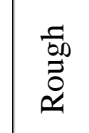 & $\begin{array}{l}\bar{\Xi} \\
\text { Oे } \\
\text { क }\end{array}$ & $\begin{array}{l}\text { 마 } \\
\overrightarrow{0} \\
\widetilde{\approx}\end{array}$ & 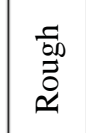 & 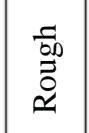 & 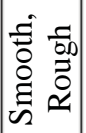 \\
\hline $\begin{array}{l}\omega \\
\tilde{m} \\
\tilde{n}\end{array}$ & $\begin{array}{l}\text { w } \\
\text { qu } \\
\text { in }\end{array}$ & $\begin{array}{l}n \\
\stackrel{2}{0} \\
\infty\end{array}$ & 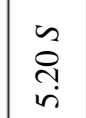 & $\begin{array}{l}\text { us } \\
\text { o }\end{array}$ & $\begin{array}{l}\tilde{a} \\
\stackrel{R}{i} \\
\text { in }\end{array}$ \\
\hline 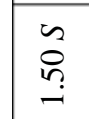 & 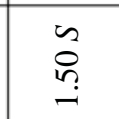 & $\begin{array}{l}n \\
\stackrel{1}{R} \\
i\end{array}$ & $\begin{array}{l}\text { w } \\
\text { டी } \\
\end{array}$ & $\begin{array}{l}\text { w } \\
\stackrel{+}{+} \\
\stackrel{\text { in }}{ }\end{array}$ & $\begin{array}{l}\text { w } \\
\infty \\
\infty \\
-\end{array}$ \\
\hline $\begin{array}{l}\text { w } \\
\stackrel{0}{\circ} \\
\stackrel{0}{-}\end{array}$ & $\begin{array}{l}\text { w } \\
\text { ڤn } \\
\text { ڤn }\end{array}$ & $\begin{array}{l}\text { w } \\
\infty \\
\stackrel{-}{\rightarrow}\end{array}$ & $\begin{array}{l}\infty \\
m \\
m \\
-\end{array}$ & $\begin{array}{l}\text { w } \\
0 \\
0 \\
-i\end{array}$ & $\begin{array}{l}\text { 足 } \\
\stackrel{\sim}{\sim} \\
\sim\end{array}$ \\
\hline & $\begin{array}{l}\text { w } \\
\text { ڤn } \\
0\end{array}$ & $\begin{array}{l}\text { w } \\
\text { ڤn } \\
\text { ڤn. }\end{array}$ & ' & $\begin{array}{ll}w & w \\
\delta & 0 \\
0 & 0 \\
0 & 0\end{array}$ & $\begin{array}{l}\frac{8}{00} \\
\text { जै के }\end{array}$ \\
\hline & & i & ' & io & 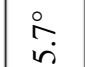 \\
\hline 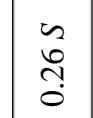 & & $\begin{array}{l}\text { w } \\
\text { ڤn } \\
\text { ڤִ }\end{array}$ & ' & ' & $\begin{array}{l}\text { us } \\
\text { hn } \\
0\end{array}$ \\
\hline $\begin{array}{l}n \\
\exists \\
\exists\end{array}$ & \begin{tabular}{l}
$\infty$ \\
\multirow{0}{0}{} \\
$\stackrel{0}{0}$
\end{tabular} & $\begin{array}{l}\infty \\
\exists \\
ت\end{array}$ & $\begin{array}{l}\infty \\
0 \\
0 \\
0\end{array}$ & ' & $\begin{array}{l}\text { u } \\
\qquad 0 \\
0 \\
0\end{array}$ \\
\hline 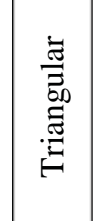 & 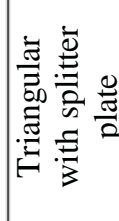 & 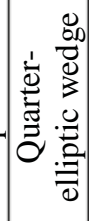 & 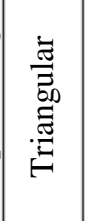 & 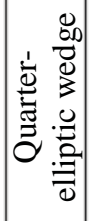 & 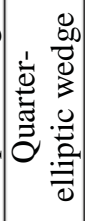 \\
\hline 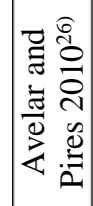 & 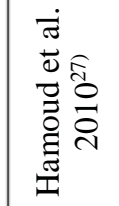 & 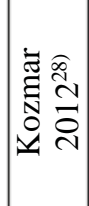 & 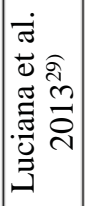 & 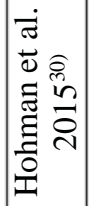 & 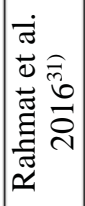 \\
\hline
\end{tabular}

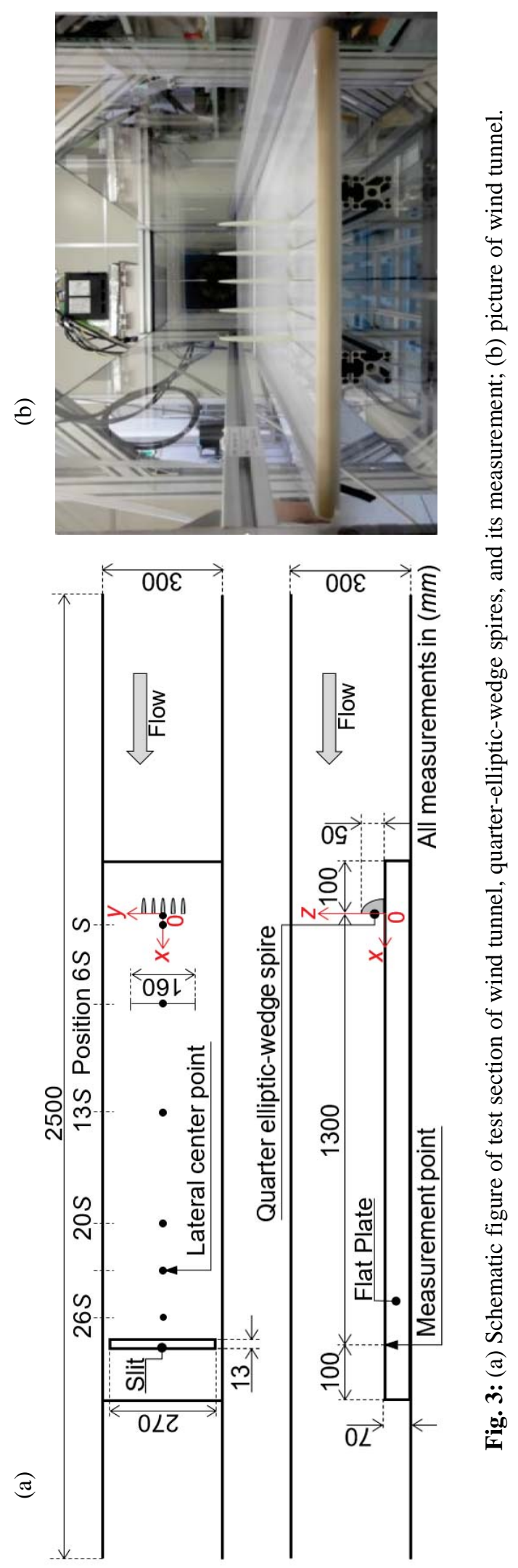


measurement of the spatial structure of the flow field past quarter-elliptic-wedge spires. To highlight the mechanism of the spires to modulate the wall boundary layer development, a simple experimental condition of the fetch area, i.e. a smooth surface, was adopted. Additional obstacles such as barriers and roughness, which have been often used to strengthen the turbulent intensity for practical use, were not used. In addition, a long streamwise fetch from up to $26 S$ was adopted to capture the transient nature of the recovery of the spires' wake and boundary layer growth. Note that there is little experimental data under the conditions of a fetch length longer than 5.4 $S$ for a smooth surface, as shown in Table 1 and Fig. 2(a).

\section{Experimental setup}

\subsection{Wind tunnel and spires}

The experiment was conducted in an open-circuit suck-through wind tunnel at the laboratory of the Interdisciplinary Graduate School of Engineering Sciences, Kyushu University, Japan. The wind tunnel has a working section of $0.3 \mathrm{~m}$ height and $0.3 \mathrm{~m}$ width with a streamwise length of $2.5 \mathrm{~m}$. The spanwise variation of the mean flow at the exit of the upwind contraction part is relatively large, with a relative standard deviation of the velocity of about $1.5 \%$ measured at a height of $10 \mathrm{~mm}$ from the tunnel floor due to the inherent effect of the contraction part and a fan. Therefore, a flat plate was installed parallel to the tunnel floor at a height of $0.07 \mathrm{~m}$ in order to newly generate a wall boundary layer. The relative standard deviation of the velocity measured at a height of 10 $\mathrm{mm}$ from the flat-plate floor was reduced to $0.7 \%$. Figs. 3(a) and 3(b) show schematic figures of the wind tunnel and a picture of the wind tunnel, respectively. Axes $x, y$, and $z$ indicate the along-wind, cross-wind (lateral), and vertical coordinate distance.

The experiment was conducted under two conditions. The first case was a control case without any spires (hereafter, the WO case). The second case used quarter-elliptic-wedge spires (hereafter, the WS case). The height, width (hereafter, $y_{s}$ ), and semi wedge angle of the spires were $0.05 \mathrm{~m}(=S), 0.005 \mathrm{~m}$ $(0.1 S)$, and $5.71^{\circ}$, respectively, as shown in Fig. 4 . The shape of the spires was determined based on Counihan $1969^{15)}$. Five spires were installed at the upwind position, $2 S$, from the leading edge of the flat plate, with a lateral interval of $0.5 \mathrm{~S}$.

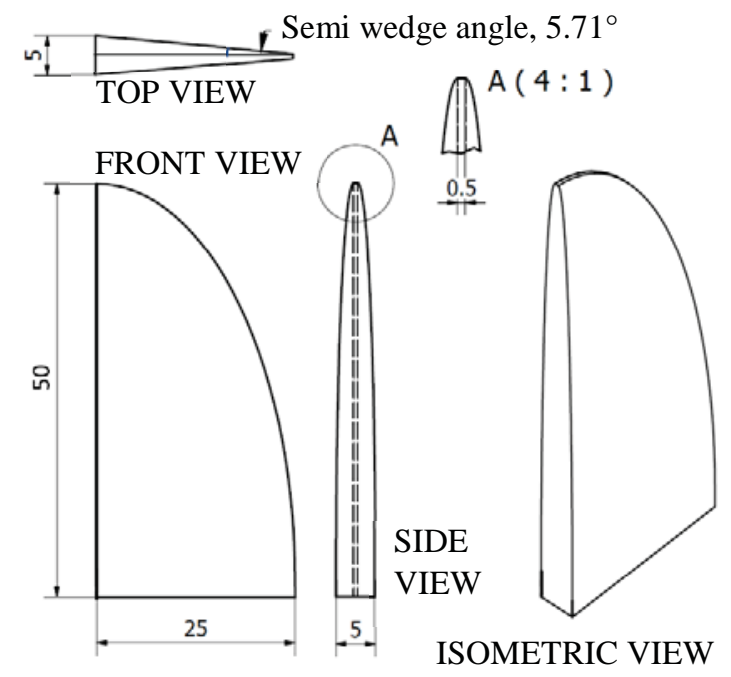

All measurements in $(\mathrm{mm})$

Fig. 4: Schematic figure of miniature of quarterelliptic-wedge spire and its dimensions.

\subsection{Measurement positions and instrumentation}

The distributions of the streamwise velocity in the lateral-vertical planes were measured at five leeward positions, from a near-wake region of $x=0.5 S$ to farwake regions of $x=6 S, 13 S, 20 S$, and $26 S$ for both the WO and WS cases. By contrast, two leeward positions ( $x=S$ and $23 S$ ) were added for the WS case. At each lateral-vertical plane, measurements were conducted at 12 different altitudes up to $z=1.2 S$ (0.06 $\mathrm{m})$ and 43 lateral positions from $y=-1.6 S(0.08 \mathrm{~m})$ to $y=1.6 S$ at intervals of $0.01 \mathrm{~m}, 0.003 \mathrm{~m}$, and $0.001 \mathrm{~m}$ spacing for $-1.6 S \leq y \leq-1 S$ and $1 S \leq y \leq 1.6 S,-1 S \leq$ $y \leq-0.04 S$ and $0.04 S \leq y \leq 1 S$, and $-0.04 S \leq y \leq 0.04$ $S$, respectively. In addition, the vertical profile of the streamwise velocity was measured at an interval of $0.002 \mathrm{~m}$ on laterally centred points $(y=0)$ at five and seven leeward positions from $x=0.002 \mathrm{~m}$ to $0.12 \mathrm{~m}$ $(2.4 \mathrm{~S})$ for the WO and WS conditions, respectively.

The streamwise velocity was measured using a single hot-wire anemometer (I-type, Kanomax, 0251R-T5) and a CTA unit (Kanomax, Model 1010). The measurement frequency and period were $1000 \mathrm{~Hz}$ and $30 \mathrm{~s}$, respectively, and the data were recorded using a data logger (Graphtec, GL900). All measurements were conducted at a reference stream velocity of approximately $10 \mathrm{~ms}^{-1}$. The calibration of the hot-wire anemometer was performed at least every $24 \mathrm{~h}$ using a pitot tube. 


\section{Results and discussion}

\subsection{Flow over a flat plate in case WO}
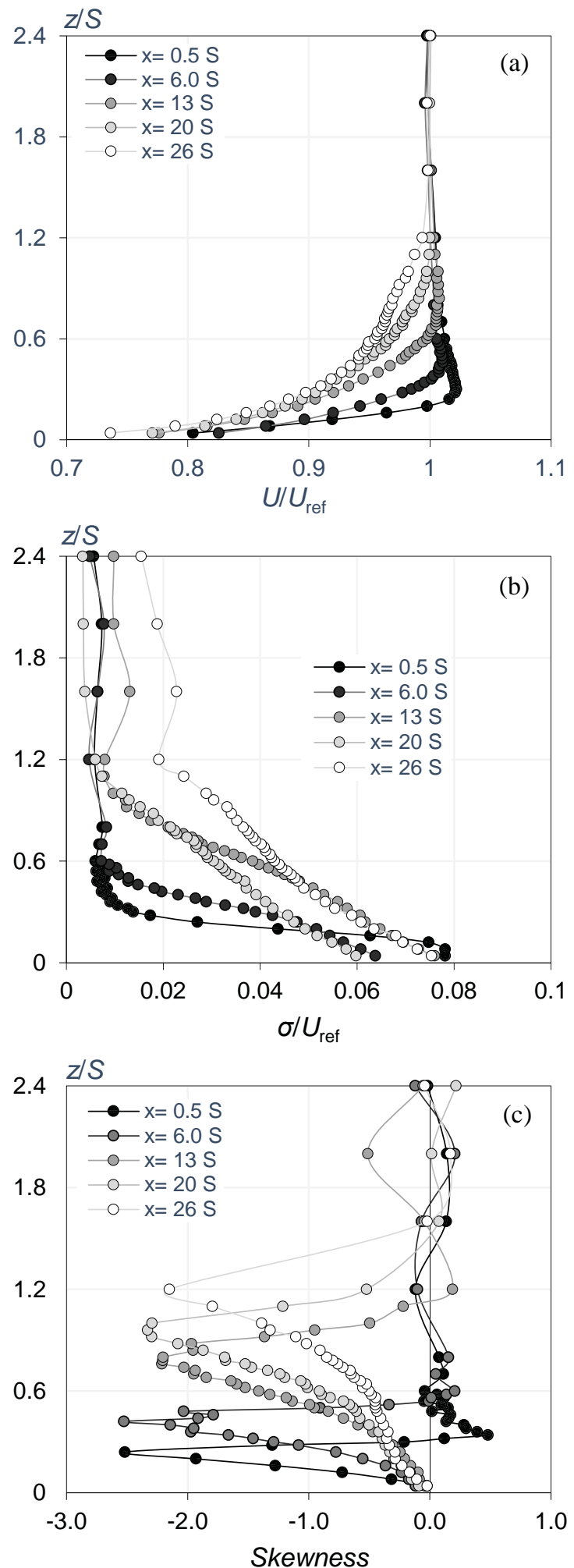

Fig. 5: Vertical velocity profiles of (a) mean, (b) standard deviation, and (c) skewness of streamwise velocity at lateral centre point $(y$ $=0$ ) of each streamwise distance $x=0.5 S, 6.0$ $S, 13 S, 20 S$, and $26 S$ for WO case. Mean and standard deviation are normalized by $U_{r e f}$, mean velocity at $y=0, z=1.6 S$, for each streamwise position.

Figure 5 shows the vertical profiles of the mean, standard deviation, and skewness of the streamwise velocity at five streamwise positions for the WO case. The mean velocity and standard deviation were normalized by $U_{\text {ref }}$, the mean velocity at $y=0$, and $z=$ 1.6 $S$ for each streamwise position.

As can be seen in Fig. 5(a), the mean wind profiles show the development of a boundary layer with the fetch length. The data of $x=0.5 S$ and $6 S$ show an inflection point at heights of around $z=0.3 S$ and 0.4 $S$, respectively, and the velocities gradually become constant at a height above $z=0.8 \mathrm{~S}$. The data of other far-wake positions show stronger velocity reduction near the wall surface up to $z=1.2 \mathrm{~S}$. The data of the standard deviation [Fig. 5(b)] at each fetch distance are large near the surface owing to strong shear, and show monotonic decrease with the height. Above $z=1.2 \mathrm{~S}$, where the mean velocity is almost height independent, the standard deviation increases slightly with the streamwise position from around $0.7 \%$ to $1.8 \%$.

By contrast, the skewness shown in Fig. 5(c) decreases with the height near the surface, shows a sharp negative peak, and returns to around zero at all streamwise positions. By comparing the profiles of the skewness with those of the mean wind, one can observe that the height of the negative peak in the skewness profile is almost consistent with the height at which the mean velocity becomes almost height independent. According to past work, i.e. Hagishima et al. 2009 ${ }^{32}$, we determined the height of the negative peak of the skewness profile as the BLH $\delta$ for the following analysis.

\subsection{Structure of flow past spires}

Figure 6 shows the distributions of the mean, standard deviation, and skewness of the streamwise velocity within a lateral-vertical plane at $x=26 S$ of the condition without a spire, i.e. the WO case. The velocity $U_{\mathrm{n}}$ is normalized by a value at $y=1.6 \mathrm{~S}, \mathrm{z}=$ $1.6 S$ for each streamwise position $U_{r e f}$, as shown in equation 1 : 


$$
\begin{aligned}
& U_{n}(x, y, z) \\
& =\frac{U(x, y, z)}{U_{\text {ref }}(x, y=1.6 S, z=1.6 S)}
\end{aligned}
$$

As can be seen in Fig. 6(a), the boundary layer with a velocity deficit owing to the wall shear undulates slightly in the lateral direction. Considering there is no obstacle at the upwind position in the WO case, the subtle lateral nonuniformity of the mean wind might be inevitably created by the contraction part of the inlet, side walls, bottom floors, and ceiling of the wind tunnel, and gradually becomes prominent with the development of the wall boundary layer. This wavy pattern of the velocity deficit corresponds to the spatial distributions of the standard deviation and skewness of $U_{n}$ in the lateral-vertical planes, as shown in Figs. 6(b) and 6(c), respectively. Both values increase as the position approaches the floor.

The distribution of the mean wind for the WS case is shown in Fig. 7 in the same manner as Fig. 6(a). The velocity is slow near the floor and behind the spires, showing five sharp peaks with elliptical shapes, especially in the near-wake region. These five wakes gradually merge and become a deep velocity-deficit layer combined with an underlying smooth wall boundary layer. In the far-wake regions at $x \geq 20 \mathrm{~S}$, the layer with a velocity deficit is relatively shallow near the laterally centre position, which might be caused by the structure of the weak secondary vortex pair in the lateral-vertical plane.

The spatial distributions of the standard deviation and skewness of $U_{n}$ in the lateral-vertical planes for the WS case at three fetch lengths are shown in Figs. 8 and 9, respectively. As expected, the regions with a strong velocity deficit coincide with layers with a large standard deviation, and the strong negative peak of the skewness is located in the borders of these regions. Irwin $1981^{16)}$ pointed out that spires with $S / 2$ lateral spacing can generate flow at a fetch distance of $6 S$ with sufficient spatial uniformity of the mean velocity. However, owing to strong turbulence created by each spire, five peaks are still observed in the present WS data at $x=6 S$, as shown in Fig. 7(c). Hohman 2015 ${ }^{30}$ ) presented similar data for the distance $5 S \leq x \leq 6 S$.

The spatially averaged BLH was calculated based on the negative skewness peak at all spanwise positions, as shown in Fig. 2(a). The data of the nearwake regions $x \leq 6 S$ indicate that the BLH of the WS case is about $32 \%$ on average larger than that of the WO case. However, one should remember that this quasi-deep wall boundary layer of the WS case contains a clear wake structure created by each spire and cannot be recognized as a uniform wall boundary layer as mentioned previously. By contrast, the BLH for $x \geq 20 S$ of the two cases are almost comparable, which means that the spire installation is not effective to deepen the boundary layer. In addition, the data at $x$ $=13 S$ of both WO and WS cases show a different trend compared to the data of $x \leq 6 S$ and $x \geq 20 S$, and the BLH of WO is larger than the other. Theoretically, the depth of naturally-grown turbulent boundary layer over a plate shows the continuous and gradual increase as the dashed line included in Fig.2
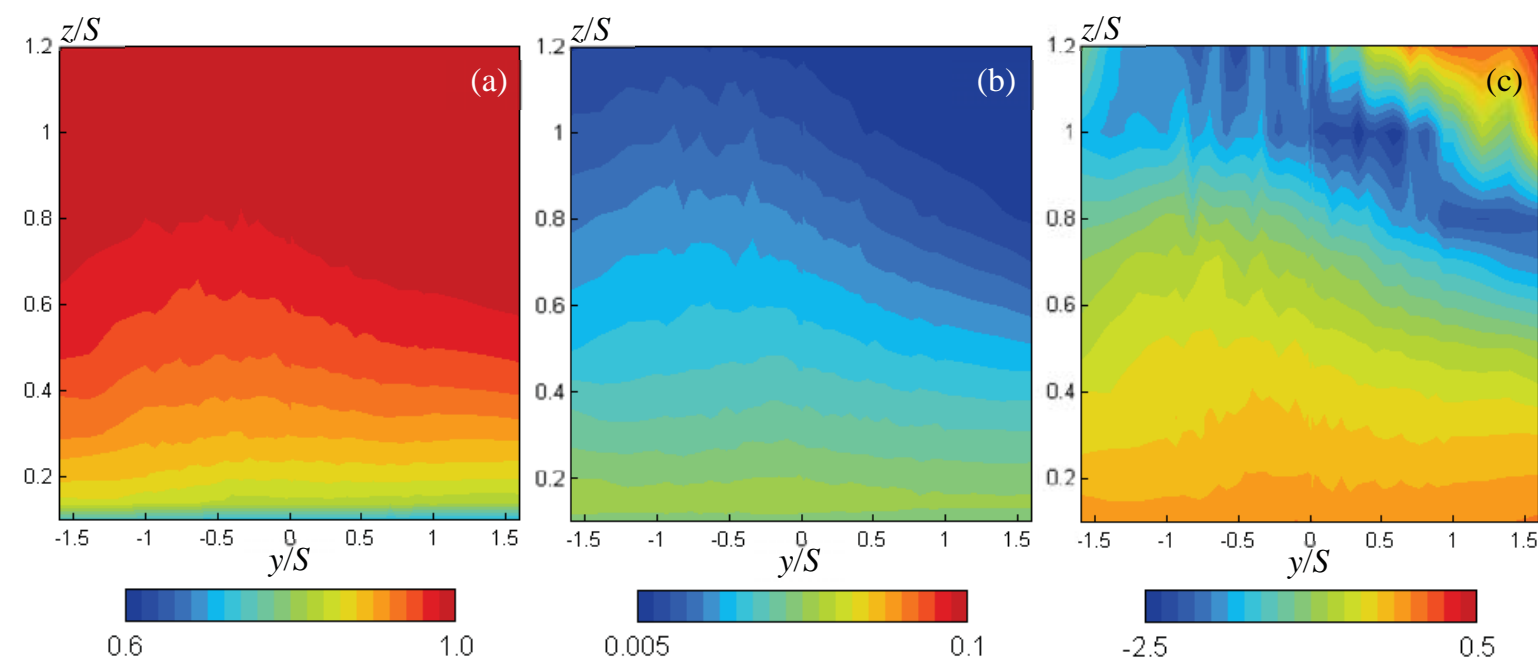

Fig. 6: Contours of (a) streamwise velocity $U_{\mathrm{n}}(x, y=1.6 \mathrm{~S}, z=1.6 \mathrm{~S})$, (b) standard deviation of streamwise velocity $U_{\mathrm{n}}(x, y=1.6 S, z=1.6 S)$, and (c) skewness of streamwise velocity $U_{\mathrm{n}}(x, y=1.6 \mathrm{~S}, z=1.6 \mathrm{~S})$ for WO case at $x=26 S$. 

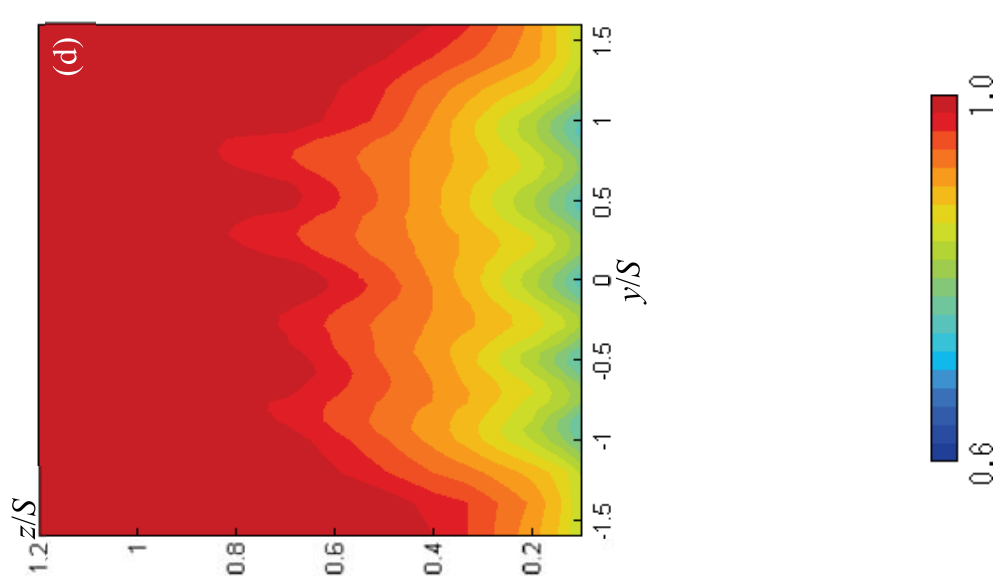

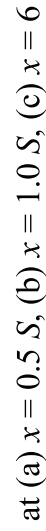
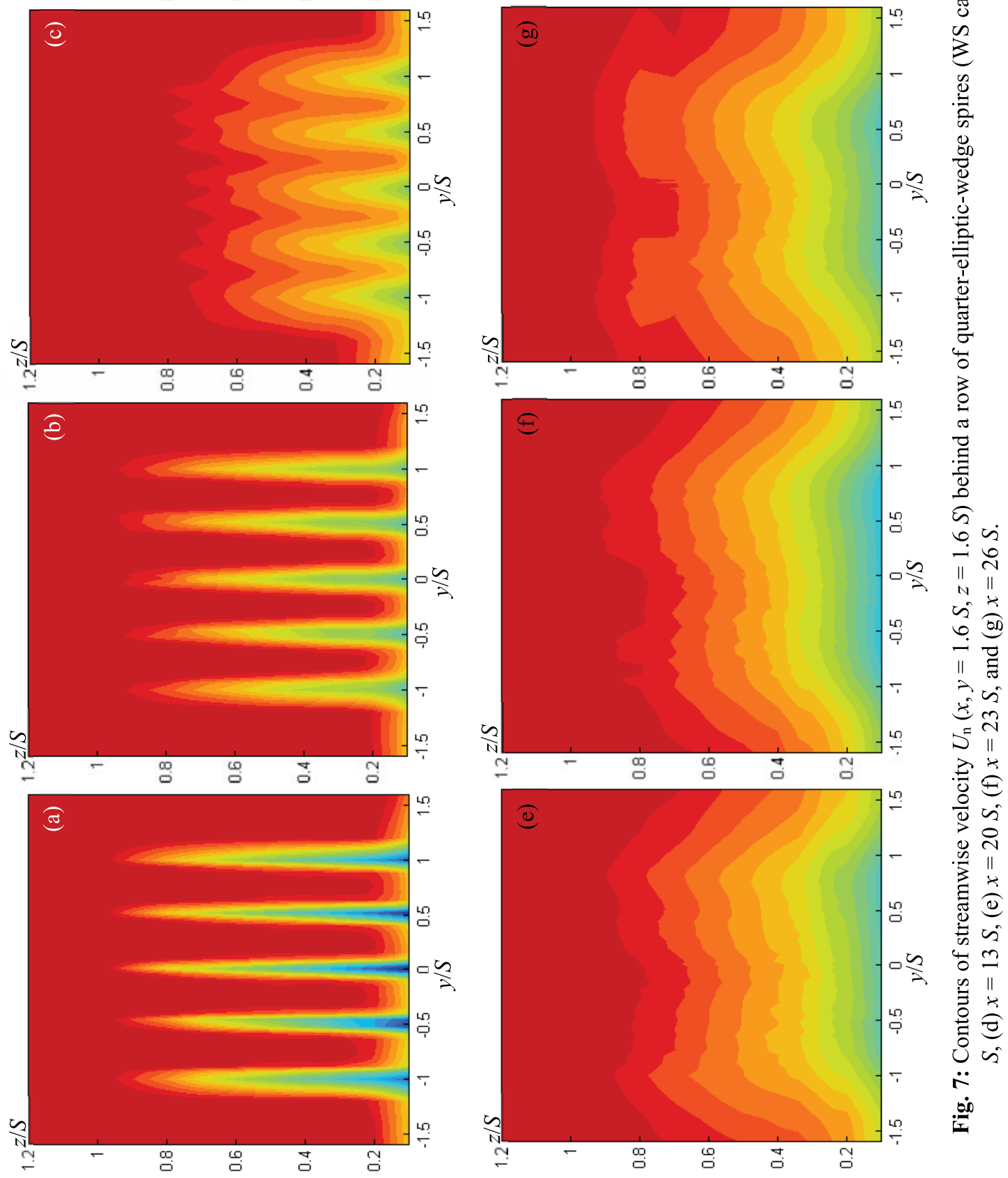

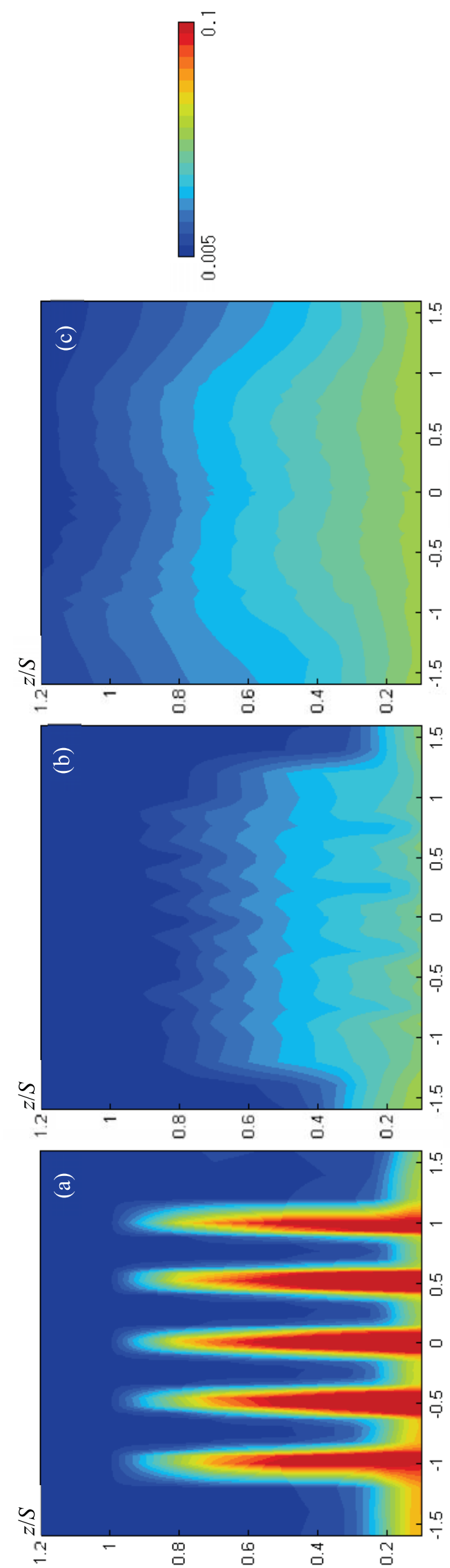

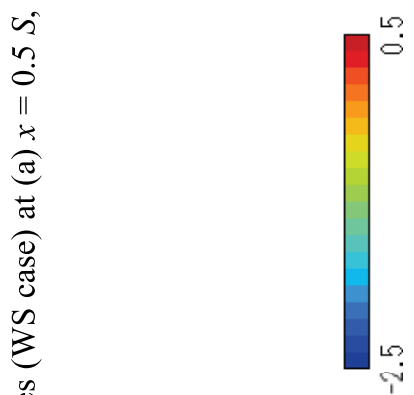

6

2
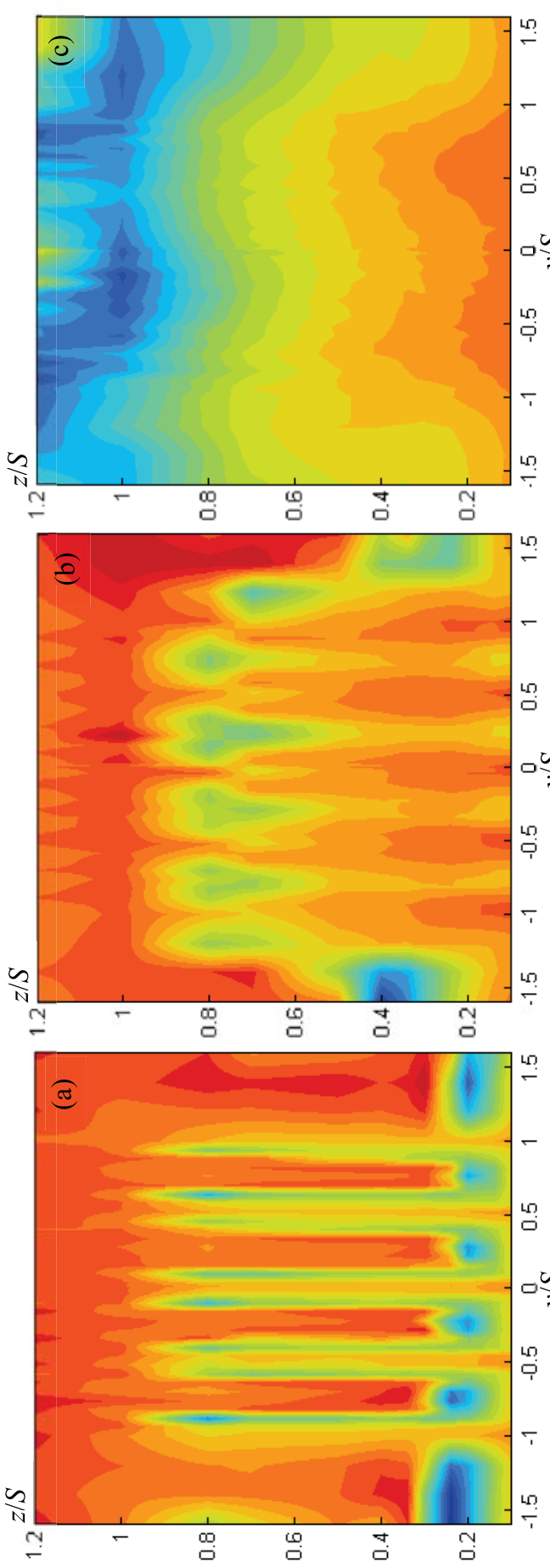

$\omega$ 
behaves. However, the present data of WO case indicate the discontinuity of the BLH growth. Since the spatial distribution of the mean, standard deviation, and skewness of the velocity within a plane of $x=13$ $S$ exhibits two clear peaks of BL (not shown here), which result in the relatively large whisker, this change of the tendency of the BLH growth might be related to the weak secondary vortices within a $\mathrm{y}-\mathrm{z}$ plane.

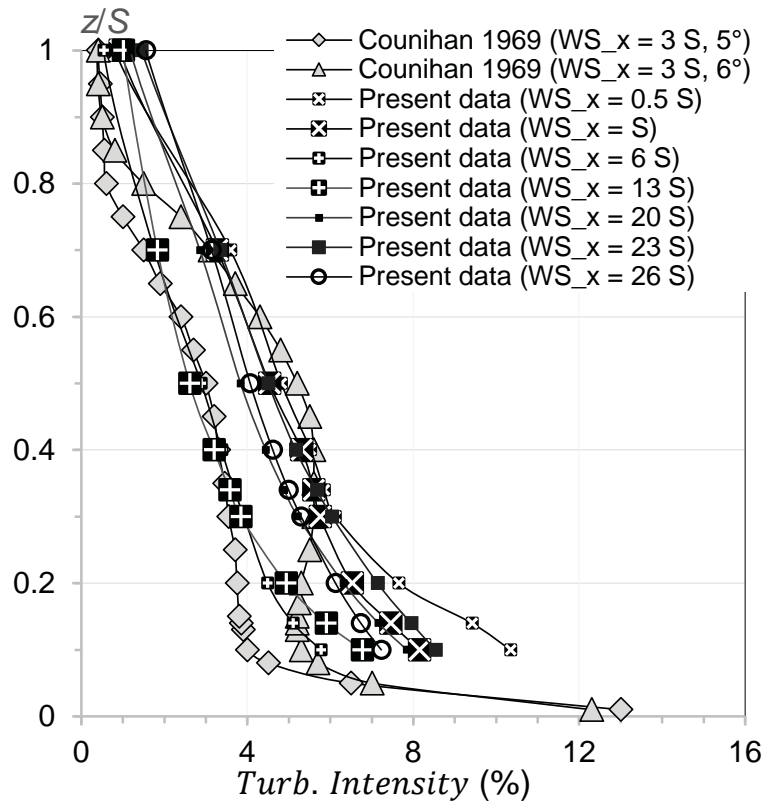

Fig. 10: Laterally averaged turbulence intensity of spires at different fetch distances. Data of Counihan $1969^{15)}$ with different semi-wedge angles of $5^{\circ}$ and $6^{\circ}$ are included

Figure 10 shows a comparison of turbulence intensities between Counihan 1969 ${ }^{15)}$ and the present data. The author excludes the data above $z=1 S$ of Counihan $1969^{15}$ ) because the data may be affected by the top wall of the wind tunnel with a height of $1.2 \mathrm{~S}$. All experiments were conducted with a lateral spire spacing of $s=0.5 \mathrm{~S}$. As shown in Fig. 10, the turbulence intensity of the present data gradually decreases with a streamwise distance of $0.5 S \leq x \leq$ $6 S$. However, farther downstream at $13 S \leq x \leq 26 S$, the data increases slightly, especially near the floor, and becomes almost consistent with $2.8 \%$ at height $z$ $=0.7 \mathrm{~S}$. Near the floor, both sets of Counihan $1969^{15)}$ data show a turbulence intensity of almost $13 \%$ that gradually decreases with height, in similar order to the present data measured at $x=6 \mathrm{~S}, \mathrm{z}=0.1 \mathrm{~S}, 0.4$ $S$, and $0.5 S$.
The standard deviation of velocity at the same $x$ and $z$ positions normalized by the average velocity in a lateral measurement line (hereafter, $\mathrm{RSD}_{U}$ ) for several selected streamwise positions is shown in Fig. 11. For reference, the WO case data measured at $x=6$ $S$ and $26 S$ are included. The data for the WO case at $x$ $=6 S$ near the floor shows a variation of $0.4 \%$, and reached its maximum variation of $1.4 \%$ at $z=0.5 \mathrm{~S}$ before gradually decreasing with the height from the floor, with $0.5 \%$ at $z=1 \mathrm{~S}$. However, the data farther downstream at $x=26 S$ has a variation of $0.4 \%$ and is much smaller except for the data near the floor at 0.1 $S \leq z \leq 0.2 S$.

Meanwhile, the data of the WS case show a decreasing trend from heights very close to above the floor and towards farther downstream. By comparing both cases at higher positions ( $z \geq 0.5 S$ ), the data of the WS case are almost consistent with those of the WO case, probably because the lateral widths of the spires decrease with height. Therefore, the heterogeneity of the mean flow near the floor is strong. The data of Counihan 1969 ${ }^{15)}$ for spires with $s=0.5 \mathrm{~S}$ at $x=3 S$ are of a similar order as the present data at $x$ $=6 S$.

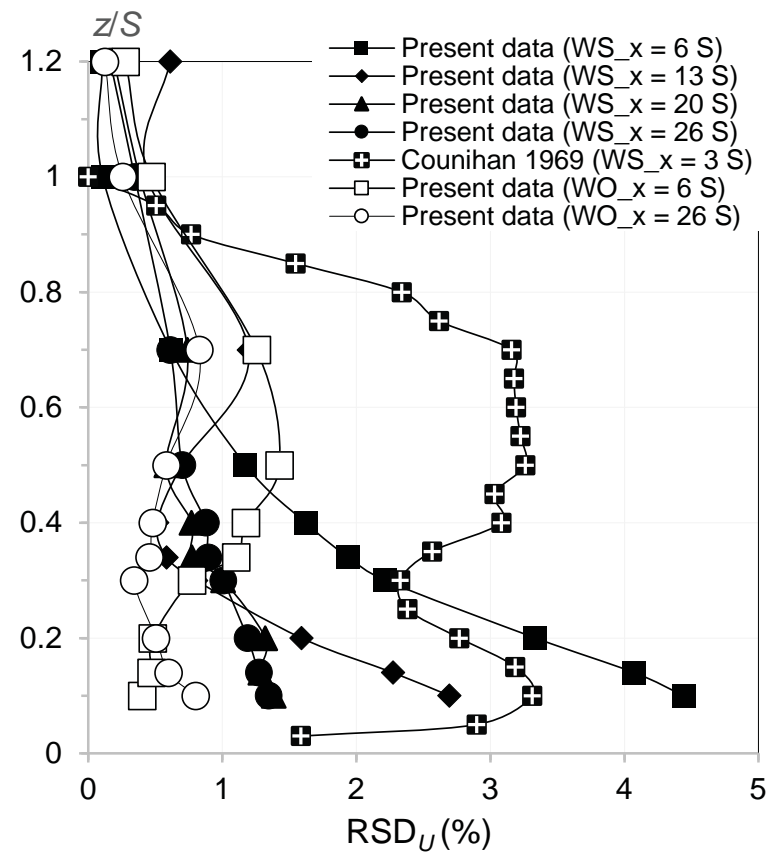

Fig. 11: Relative standard deviation $\mathrm{RSD}_{U}$ (\%) with both present data of WO and WS cases at several chosen streamwise distances. Counihan $1969^{15)}$ data measured at $x=3 S$ is also included. All experimental measurements with spires lateral spacing $s=$ $0.5 S$. 
Figure 12 shows $\mathrm{RSD}_{U}$ against a fetch length normalized with a spire height of $x / S$. The past experiments of Armitt and Counihan 1968 14 and Hohman et al. 2015 ${ }^{30}$ are also included for comparison. The data for the WO case at height $z=0.5$ $S$ show a variation of less than $1 \%$ and a range between $0.2 \%-0.7 \%$, except at $x=6 \mathrm{~S}$. On the other hand, the WO data at height $z=1 S$ show a variation of less than $1 \%$ between $0.1 \%-0.4 \%$ at all streamwise positions, which is supposed to be the original condition of this wind tunnel.

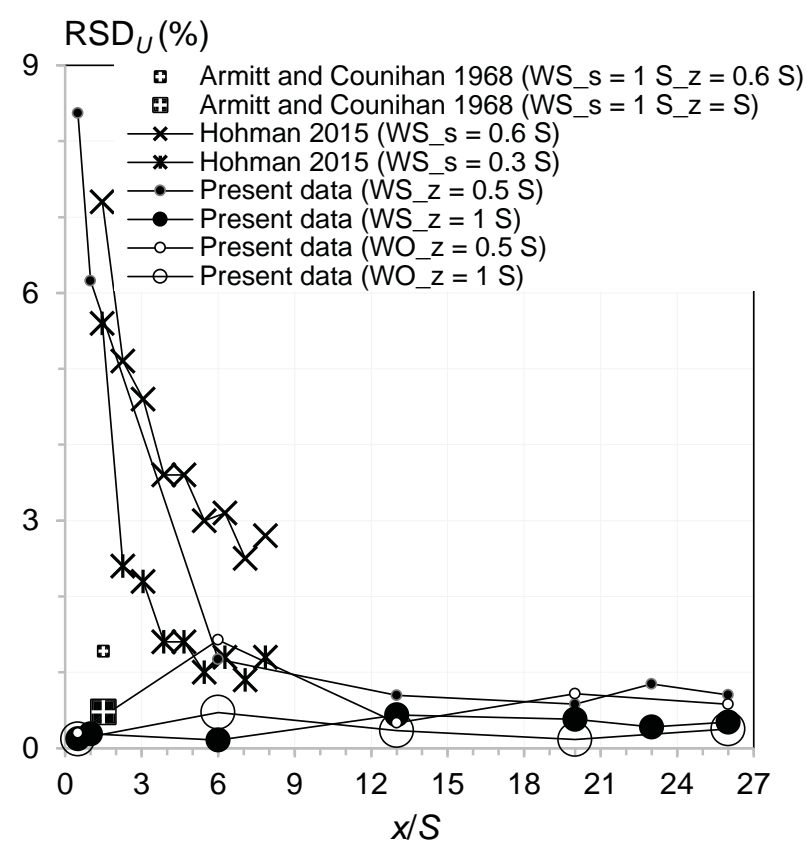

Fig. 12: Standard deviation of streamwise velocity $U_{\mathrm{n}}$ normalized by laterally averaged $U_{\mathrm{n}}$ at several chosen heights against streamwise distance. Lateral-spire spacing used in Armitt and Counihan $1968^{14)}$ is $s=1 S$. Hohman $2015^{30)}$ compared with and without barriers with different lateral-spire spacing, i.e. without barrier for $s=0.6 S$ and with peaksaligned barrier for $s=0.3 S$.

By contrast, the data of the WS case at $z=0.5 \mathrm{~S}$ show a rapid reduction from $8.4 \%$ in the near-wake region to $1.2 \%$ at $x=6 S$, and gradually decrease to $0.7 \%$ at $x \geq 13 S$. In addition, the WS data at $z=S$ show a variation of $0.1 \%$ to $0.4 \%$ at all streamwise positions, which is consistent with the WO data at $z=$ S. Counihan 1969 $9^{15)}$ claimed that the spatial heterogeneity is adequate when on the order of $1 \%$ at any given height. By contrast, the present data suggest that a uniform boundary layer flow with less than $1 \%$ spanwise variation of the mean flow would be obtained at $x \geq 13 S$. However, most past studies used relatively short-length wind tunnels (an exception would be Castro 197920). To generate suitable uniformity of flow, several countermeasures such as a smaller lateral spacing of spires as proposed by Hohman et al. 2015 ${ }^{30}$, the installation of roughness in the fetch area, and use of a barrier should be considered.

\section{Conclusions}

The present paper provides a comprehensive review of past studies on the flow behind spires, and clarifies the current issue of the spire setting in a wind tunnel experiment. In addition, the authors investigated the spatial structures of flow past a row of quarter-elliptic-wedge spires over a smooth wall with a streamwise length of $26 \mathrm{~S}$, which is much longer than the fetch length of the former studies, by using hotwire anemometry. Furthermore, the streamwise distance required for suitable uniformity of the flow was discussed. From the present experimental results, $x=13 S$ is the least streamwise distance required for the wake of elliptic-wedge spires to generate a naturally developed smooth wall boundary layer with less than $1 \%$ lateral heterogeneity.

\section{References}

1) United Nations, Department of Economic and Social Affairs, Population Division, The world's cities in $2016 \quad-$ Data Booklet (ST/ESA/SER.A/392) (2016).

2) Oke, T. R. Boundary layer climates, $2^{\text {nd }}$ ed., Londong Metheun, 435 (1987).

3) Blocken, B., and Carmeliet, J. Pedestrian wind environment around buildings: literature review and practical examples, J. of Thermal Env. \& Bldg. Sci., vol. 28, no. 2 (2004).

4) Grimmond, C. S. B. Progress in measuring and observing the urban atmosphere, Theor. Appl. Climatol., no. 84, pp. 3-22 (2006).

5) Kanda, M. Progress in the scale modelling of urban climate: Review, Theor. Appl. Climatol., no. 84, pp. 23-33 (2006).

6) Aly, M. A. Atmospheric boundary-layer simulation for the built environment: past, present and future, Building and Environment, no. 75, pp. 206-221 (2014).

7) Stull, R. B. An introduction to boundary layer meteorology, Kluwer Academic Publishers, Dordrecht (1988). 
8) Cermak, J. E., and Arya, S. P. S. Boundary layer meteorology 1, pp. 40-60 (1970).

9) Counihan, J. Simulation of an adiabatic urban boundary layer in a wind tunnel, Atmospheric Environment Pergamon Press, vol. 7, pp. 673-689 (1973).

10) Nishi, A., Kikugawa, H., Matsuda, Y., and Tashiro, D. Turbulence control in multiple-fan wind tunnels, J. of Wind Eng. Ind. Aerodyn., no. 67\&68, pp. 861-872 (1997).

11) Elder, J. W. Steady flow through non-uniform gauzes of arbitrary shape, J. Fluid Mech., vol. 5, pp. 355-368 (1959).

12) Hunt, J. C. R. and Fernholz, H. Wind tunnel simulation of the atmospheric boundary layer: a report on Euromech 50, J. Fluid Mech., vol. 70, pp. 543-559 (1975).

13) Guimarães, J. H. D., dos Santos, S. J. F., Jr., Su, J., and Silva Freire, A. P. Large artificially generated turbulent boundary layers for the study of atmospheric flows, Proceedings of the $15^{\text {th }}$ Brazilian Congress of Mechanical Engineering (COBEM99) (1999).

14) Armitt, J., and Counihan, J. The simulation of the atmospheric boundary layer in a wind tunnel, J. of Atmospheric Environment, vol. 2, pp. 49-71, (1968).

15) Counihan, J. An improved method of simulating an atmospheric boundary layer in a wind tunnel, Atmospheric Environment Pergamon Press, vol. 3, pp. 197-214 (1969).

16) Irwin, H. P. A. H. The design of spires for wind simulation, J. of Wind Eng. Ind. Aerodyn., vol. 7, pp. 361-366 (1981).

17) Counihan, J. Wakes behind two-dimensional surface obstacles in turbulent boundary layers, $J$. Fluid Mech., vol. 64, part 3, pp. 529-563 (1974).

18) Standen, N. M. A spire array for generating thick turbulent shear layers for natural wind simulation in wind tunnels, National Research Council of Canada, NAE Rep., LTR-LA-94 (1972).

19) Cook, N. J. Wind tunnel simulation of the adiabatic atmospheric boundary layer by roughness, barrier and mixing-device methods, $J$. of Industrial Aerodynamics, vol. 3, pp. 157-176 (1978).

20) Castro, I. P., Relaxing wakes behind surfacemounted obstacles in rough wall boundary layers, J. Fluid Mech., vol. 93, part 4, pp. 631-659 (1979).

21) Meroney, N. R., Pavageau, M., Rafailidis, S., and Schatzmann, M., Study of line source characteristics for 2-D physical modelling of pollutant dispersion in street canyons, $J$. of Wind Eng. Ind. Aerodyn., vol. 62, pp. 37-56 (1996).

22) Pavageau, M., and Schatzmnn, M., Wind tunnel measurements of concentration fluctuations in an urban street canyon, Atmospheric Environment, vol. 33, pp. 3961-3971 (1999).

23) Farell, C., and Iyengar, A. K. S. Experiments on the wind tunnel simulation of atmospheric boundary layers, J. of Wind Eng. Ind. Aerodyn., vol. 79, pp. 11-35 (1999).

24) Iyengar, A. K. S., and Farell, C. Experimental issues in atmospheric boundary layer simulations: Roughness length and integral length scale determination, J. of Wind Eng. Ind. Aerodyn., vol. 89, pp. 1059-1080 (2001).

25) Balendra, T., Shah, D. A., Tey, K. L., and Kong, S. K. Evaluation of flow characteristics in the NUS-DHB wind tunnel, J. of Wind Eng. Ind. Aerodyn., vol. 90, pp. 675-688 (2002).

26) Avelar, A. C. and Pires, L. B. M. Simulation of the atmospheric boundary layer in a closed-circuit wind tunnel with short test section, $27^{\text {th }}$ AIAA Aerodynamic Measurement Technology and Ground Testing Conference (28 June-1 July 2010).

27) Hamoud A. A. N., Ali K. A. R., Abd. E. M. N., and Hamdy M. S., Design and construction of a wind tunnel for environmental flow studies, J. of Engineering Sciences, Assiut University, vol. 38, no. 1, pp. 177-193 (2010).

28) Kozmar, H. Improved experimental simulation of wind characteristics around tall buildings, J. of Aerospace Engineering (ASCE), vol. 25, pp. 670679 (2012).

29) Luciana, B. M. P., Igor, B. de Paula, Gilberto, F., Ralf, G., and Roberto, M. G. Simulations of the atmospheric boundary layer in a wind tunnel with short test section, J. Aerosp. Technol. Manag., vol. 5, no. 3, pp. 305-314 (2013).

30) Hohman, T. C., Van Buren, T., Martinelli, L., and Smits, A. J. Generating an artificially thickened boundary layer to simulate the neutral atmospheric boundary layer, J. of Wind Eng. Ind. Aerodyn., vol. 145, pp. 1-16 (2015).

31) Rahmat, N. A., Hagishima, A., Ikegaya, N., and Tanimoto, J., An experimental study on aerodynamic interaction between a boundary layer generated by a smooth and rough wall and a wake behind a spire, Engineering Sciences Reports, Kyushu University, vol. 37, no. 2, pp. 19-26 (2016). 
32) Hagishima, A., et al. Aerodynamic parameters of regular arrays of rectangular blocks with various geometries, Boundary Layer Meteorol., vol. 132, pp. 315-337 (2009). 